\title{
From spectra to atmospheres: solving the underconstrained retrieval problem for exoplanets
}

\author{
Joanna K. Barstow ${ }^{1}$, Suzanne Aigrain ${ }^{1}$, Patrick G. J. Irwin ${ }^{1}$, Neil \\ Bowles $^{1}$, Leigh N. Fletcher ${ }^{1}$ and Jae-Min Lee ${ }^{2}$ \\ ${ }^{1}$ Department of Physics, University of Oxford, Oxford, UK \\ email: jo.barstow@astro.ox.ac.uk \\ ${ }^{2}$ Institute for Theoretical Physics, University of Zürich, Zürich, Switzerland
}

\begin{abstract}
Spectroscopic observations of transiting exoplanets have provided the first indications of their atmospheric structure and composition. Optimal estimation retrievals have been successfully applied to solar system planets to determine the temperature, composition and aerosol properties of their atmospheres, and have recently been applied to exoplanets. We show the effectiveness of the technique when combined with simulated observations from the proposed space telescope EChO, and also discuss the difficulty of constraining a complex system with sparse data and large uncertainties, using the super-Earth GJ 1214b as an example.
\end{abstract}

Keywords. radiative transfer, methods: data analysis, planets and satellites: individual

\section{Introduction}

When a planet passes in front of its parent star as seen from the Earth, the resultant reduction in the amount of observed starlight varies as a function of wavelength, depending on the absorptive properties of the planets atmosphere; when the planet is eclipsed by its parent star, a difference measurement can yield its emission/reflection spectrum. However, a lack of a priori knowledge about the planets bulk properties, cloudiness and temperature can make it difficult to find a unique solution. The NEMESIS radiative transfer and retrieval tool (Irwin et al. 2008) allows this process to be explored. Barstow et al. (2013a) use NEMESIS to assess the feasibility of constraining exoplanet atmospheres with EChO (Tinetti et al. 2012), a proposed ESA space telescope designed to observe continuous transmission and eclipse spectra of transiting extrasolar planets between 0.55 and $16 \mu \mathrm{m}$.

\section{GJ 1214b: current observations}

GJ 1214b (Charbonneau et al. 2009) is a super-Earth transiting an M dwarf 13 pc away. Despite measurements of transmission through its atmosphere in the visible and infrared from a range of space- and ground-based sources (e.g. Bean et al. 2011), little can be inferred about the atmosphere. The flat transmission spectrum could be evidence of an atmosphere with a high molecular weight, but could also indicate a $\mathrm{H}_{2}-\mathrm{He}$ atmosphere with high altitude haze or cloud, and the current data are shown to have insufficient signal-to-noise and wavelength coverage to discriminate between these scenarios (Barstow et al. $2013 \mathrm{~b}$ and references therein). 


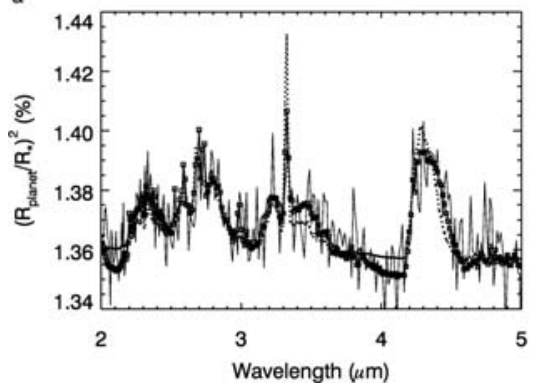

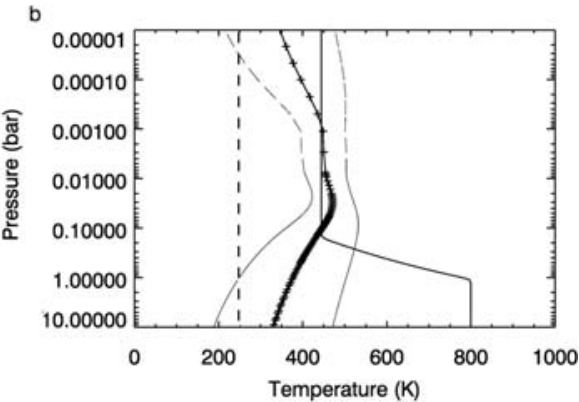

Figure 1. a) The GJ 1214b noisy synthetic transmission spectrum (thin line) for a $50 \% \mathrm{H}_{2} \mathrm{O}$ atmosphere is fit better by a $50 \% \mathrm{H}_{2} \mathrm{O}$ model (black line with points) than by a $\mathrm{H}_{2}$-He model (dotted line). This is particularly evident in the $\mathrm{CH}_{4}$ band at $3.3 \mu \mathrm{m}$ and the $\mathrm{CO}$ band at $4.3 \mu \mathrm{m}$. b) Input temperature (black line) a priori temperature (dashed line) and retrieved temperature (crosses) with error (fine dashed lines) for a secondary eclipse retrieval. The correct stratospheric temperature is retrieved but there is no information about the deep atmosphere temperature.

\section{GJ 1214b with EChO}

Barstow et al. (2013a) generate a series of model hot Jovians, using NEMESIS as a forward model to calculate noisy synthetic transmission and eclipse spectra of these, and perform retrieval feasibility tests for EChO observations. Barstow et al. (2013b) use a similar technique to demonstrate that, by observing 30 transits of GJ 1214b with EChO, a high molecular weight atmospheric scenario would be excluded if the planet in fact has a cloudy extended atmosphere. Using the same technique and model parameters, we show that if the atmosphere instead contained $50 \%$ water vapour, 60 transits would be required to achieve the requisite level of signal and distinguish between the two scenarios. When we add the noise expected for 60 observations to the best fit $\mathrm{H}_{2} \mathrm{O}$-dominated model from Barstow et al. (2013b) and fit it with a $50 \% \mathrm{H}_{2} \mathrm{O}$ model atmosphere, the reduced- $\chi^{2}$ parameter is 1.05 , as opposed to 1.31 if it is fit with a cloudy $\mathrm{H}_{2}$-He model (Figure 1a shows the fit between 2 and $5 \mu \mathrm{m}$ ). All models are as in Barstow et al. (2013b), to which we refer the reader for details. An emission spectrum of GJ 1214b could be obtained with 30 secondary eclipse observations, allowing an observational constraint to be placed on the stratospheric temperature of the planet for the first time and providing information about the albedo and therefore cloudiness of the planet (Figure 1b). EChO will be a valuable mission for the study of transiting extrasolar planets, and space-based spectroscopy over a large wavelength range is important for detailed study of planets like GJ 1214b. Optimal estimation retrieval is shown to be a powerful technique for analysing these data.

\section{References}

Barstow J. K., Aigrain S., Irwin P. G. J., Bowles N., Fletcher L. N., \& Lee J.-M. 2013, MNRAS, 430,1188

Barstow J. K., Aigrain S., Irwin P. G. J., Fletcher L. N., \& Lee J.-M. 2013, MNRAS, 434, 2616

Bean J. L., Désert J.-M., Kabath P., Stalder B., Seager S., Miller-Ricci Kempton E., Berta Z. K., Homeier D., Walsh S., \& Seifahrt A., 2011 ApJ, 743, 92

Charbonneau D., Berta Z. K., Irwin J., Burke C. J., Nutzman P., Buchhave L. A., Lovis C., Bonfils X., Latham D. W., Udry S., Murray-Clay R. A., Holman M. J., Falco E. E., Winn J. N., Queloz D., Pepe F., Mayor M., Delfosse X., \& Forveille T. 2009, Nature, 462, 891

Irwin P. G. J., Teanby N. A., de Kok R., Fletcher L. N., Howett C. J. A., Tsang C. C. C., Wilson C. F., Calcutt S. B., Nixon C. A., \& Parrish P. D. 2008, JQSRT, 109, 1136

Tinetti G., et al., 2012, Exp. Astr., 34, 311 